\title{
Using A Digital Temperature Sensor To Measure The Temperature Develop- ment During A Polishing Process
}

\author{
Benisch Michael ${ }^{1, *}$, Bogner Werner ${ }^{1, * *}$, and Rascher Rolf ${ }^{1, * * *}$ \\ ${ }^{1}$ Technische Hochschule Deggendorf, Dieter-Görlitz-Platz 1, 94469 Deggendorf
}

\begin{abstract}
The temperature development within a lens is recorded during processing with a digital temperature sensor. The development of the temperature over time shows dependencies on the relative speed and the contact pressure on the polishing wheel. Furthermore, the amount of polishing suspension flowing into the polishing gap may have an influence on the temperature development.
\end{abstract}

\section{Introduction}

The temperature, generated by a process contributes to the energy, which is caused by mechanical friction or chemical processes [1]. Therefore, the measurement of the temperature gives a hint on the energy induced in the process. The temperature change (increase or decrease) is measured during a common polishing process.

\section{Experimental Setup}

The experimenal setup consists of three components:

- Polishing wheel, with a stepwise (0 to 10 ) adjustable rotation speed between $201 / \mathrm{min}$ to $9001 / \mathrm{min}$.

- Polishing suspension (Hastilite PO, uncooled)

- Lens with integrated temperature sensor (SHT31)

Different experiments were carried out, including the measurement of the influence of

- Relative velocity

- Pressure and pressure variations during process

- High pressure and maximum possible velocity

to the temperature that adjusts itself in the lens. The lenses prepared for the experiment can be described as "smart" workpieces. They allow conclusions to be drawn from polishing influences on the resulting temperature. The resulting temperature corresponds to the energy input caused by friction and chemical effects. In a pure mechanical system, the source of heat are the pad-workpiece friction and material removal [1].

\subsection{Digital Temperature Sensor}

The absolute accuracy of the sensor is $\pm 0.2 \mathrm{~K}$, the repeatability can be chosen manually and is between $\pm 0.2 \mathrm{~K}$ to $\pm 0.04 \mathrm{~K}$. Usually their value is $\pm 0.08 \mathrm{~K}$ (setting for the experiments). The evaluation takes place via an Arduino Uno R3 microcontroller board, which is connected to the sensor via I2C communication protocol.

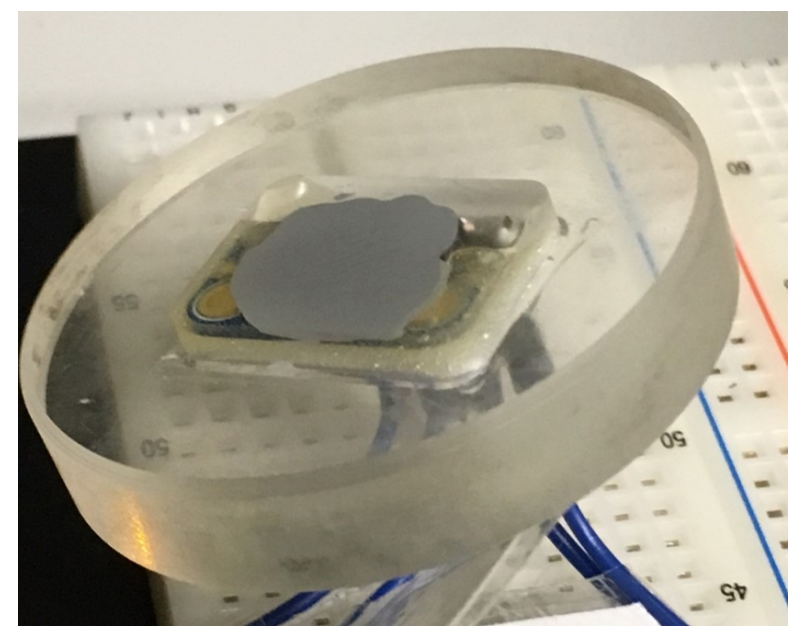

Figure 1: Glass sample with integrated digital temperature sensor. The grey area represents the conductive paste between the glass and the sensor. The dimensions of the sensor are only $2 \mathrm{~mm} \times 2 \mathrm{~mm}$.

The sensor is glued into a milled recess in the lens. The thermal connection to the glass material is made by an electrically non-conductive heat conducting paste. The sensor's optional humidity detection feature was not used during the experiments.

\subsection{Proof of principle: Maximum heating}

This test was carried out to cause a maximum possible heating of the lens. For this purpose, the workpiece was

\footnotetext{
*e-mail: michael.benisch@th-deg.de

**e-mail: werner.bogner@th-deg.de

***e-mail: rolf.rascher@th-deg.de
} 


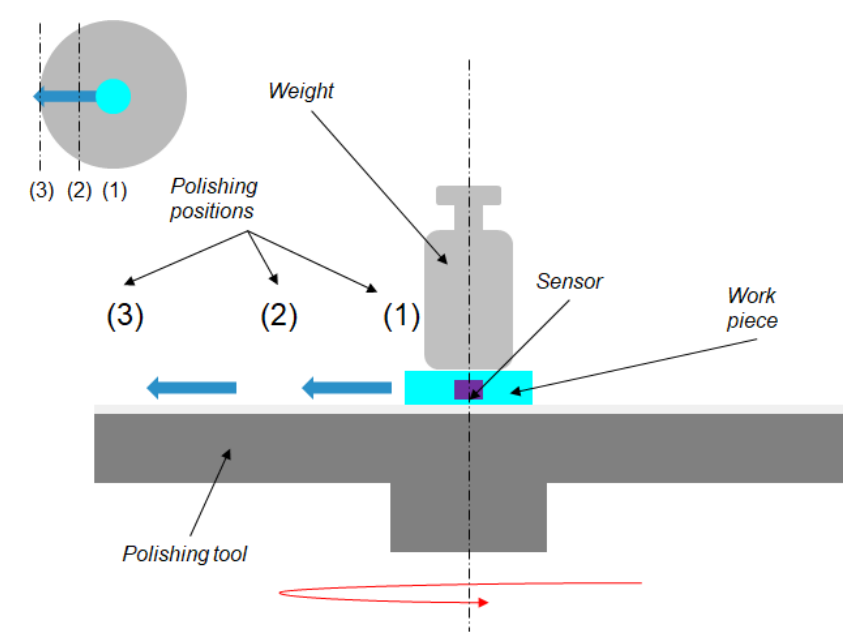

Figure 2: Arrangement of the experiment. The polishing at various points changes the relative speed between tool and workpiece. The weight acts as a force from above on the workpiece equipped with a sensor.

polished at maximum rotation speed $9001 / \mathrm{min}$ and with a high contact pressure at the edge of the polishing wheel in order to achieve a maximum relative velocity between workpiece and tool. The pressing force was introduced by a weight of $2 \mathrm{~kg}$ and a manual guidance of the workpiece.

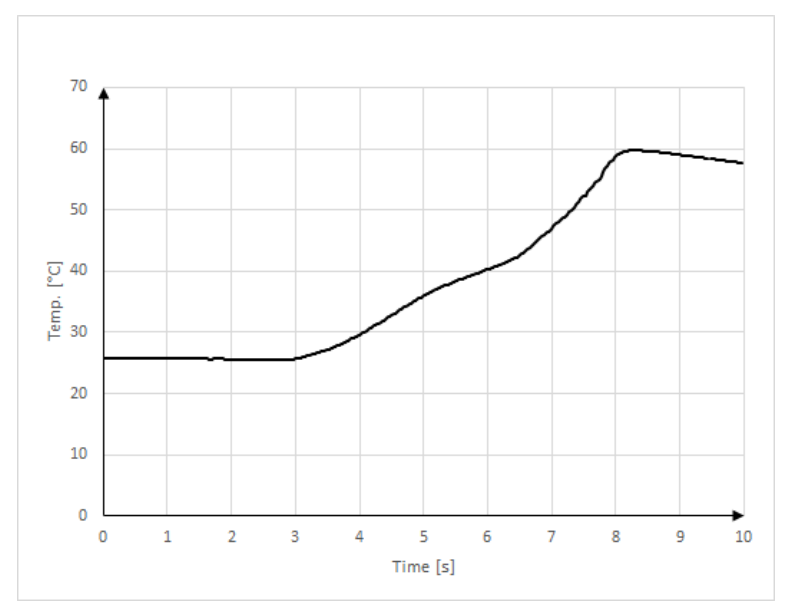

Figure 3: Temperature development of the glass lens shown in figure 1 with approximately constant high pressure and maximum adjustable speed of the polishing wheel. The highest reached value is almost $60^{\circ} \mathrm{C}$. At this point, the experiment was stopped to prevent damage to the polishing wheel. Immediately after finishing the polishing process, a cooling of the workpiece can be observed.
The measurement shows, that the lens can reach a temperature of almost $60{ }^{\circ} \mathrm{C}$ under the conditions of a high speed and high pressure process without cooling. It also shows, that cooling starts instantly after ending the processing.

The next experiments will focus on machining at variable speeds and pressures under process conditions (integration of a supply and cooling system for the polishing slurry). The part of mechanical and chemical interactions will be investigated during processing. Especially chemical processes are accelerated or slowed down by the influence of temperature. This presumed influence is to be proven.

The impact of an insufficient supply of polishing suspension is also considered. In addition the division of temperature, which occurs between workpiece, tool and slurry [2] will also be considered by the integration of further sensors close to the process.

\section{References}

[1] Evans, C. J.; Paul, E.; Dornfeld, D.; Lucca, D. A.; Byrne, G.; Tricard, M.; Klocke, F.; Dambon, O.; Mullany, B. A., Journal CIRP Annals 52 (2), 611-633 (2003)

[2] Kim, H.J; Kim, H.Y; Jeong, H.D; Lee, E.S; Shin, Y.J, Journal Journal of Materials Processing Technology, 130-131 (2002) 